\title{
Timed sequential analysis of creatine kinase in the diagnosis of myocardial infarction in patients over 65 years of age
}

\author{
P Chattington, D Clarke, W D Neithercut
}

\begin{abstract}
Aim-To assess the value of timed sequential analysis of creatine kinase (CK) activity for the early diagnosis of acute myocardial infarction (AMI) in patients over 65 years of age.

Method-Samples were collected on admission and eight to 12 hours later from 156 patients over 65 years of age. Routine cardiac enzyme activities were determined and serial electrocardiograms (ECGs) recorded. The predictive value of timed samples for $\mathrm{CK}$ activity, standard cardiac enzyme activities, and ECGs was compared with the final diagnosis on discharge.

Results-Forty one patients had a discharge diagnosis of AMI, 83 of angina pectoris, and the remaining 32 patients had other diagnoses. Electrocardiograms had a sensitivity of $55 \%$ and a specificity of $96 \%$, giving a predictive value of $86 \%$ for a negative and $84 \%$ for a positive ECG. Standard cardiac enzymes had a predictive value of $99 \%$ for a negative result but only $68 \%$ for a positive result. The logarithm of the rate of change of CK activity had a predictive value of $97 \%$ for a negative result and $95 \%$ for a positive result.

Conclusion-This study has shown that slope analysis of $\mathrm{CK}$ activity can be used for the early diagnosis of AMI in patients over 65 years of age, and that this was not affected by the presence of possible confounding diagnoses.
\end{abstract}

(F Clin Pathol 1994;47:995-998)

The rapid confirmation of a diagnosis of acute myocardial infarction (AMI) is desirable for the efficient clinical management of patients and resources. Standard cardiac enzymes collected over three days after admission to hospital provide a retrospective diagnosis but do not influence immediate patient management.

Measurement of timed sequential creatine kinase (CK) activity in serum has been proposed as one method for early confirmation of a diagnosis of AMI. ${ }^{1-6}$ Like other tests for the diagnosis of $A M I$, this test has been evaluated in patients admitted to a coronary care unit, ${ }^{12}$ however, only small numbers of patients were included in these early studies. Other investigators who studied slightly larger groups of patients found that the test gave false negative results and could not be used to exclude reliably a diagnosis of AMI. ${ }^{78}$ Although a coronary care unit provides a well controlled environment in which to conduct the evaluation of a test requiring carefully timed samples, the populations studied so far have been preselected with a high incidence of AMI, up to $55 \%, 9$ and other possible confounding diagnoses may have been excluded. As a result, the test's performance may not be as accurate when evaluated in unselected elderly subjects presenting with acute chest pain. This group of subjects may also have other potential confounding diagnoses not found in younger age groups.

The predictive value of a test improves with selection of the population studied and collection of samples under well controlled conditions. We therefore investigated the value of this test in patients over 65 years of age admitted to a busy district general hospital with acute chest pain.

Over five months, 156 patients (88 women) presenting with acute chest pain were studied. All were over 65 years of age. The time of onset of chest pain in relation to the time of admission was recorded. Three serial electrocardiograms (ECGs) were collected, standard cardiac enzyme activities comprising total CK and lactate dehydrogenase $(\mathrm{LDH})$ were determined on the three days following admission, and the results recorded. The final diagnosis at the time of discharge was also recorded.

The study was approved by the District Ethics Committee and all patients gave informed consent.

\section{Methods}

Blood samples for timed CK analysis were collected on admission and eight to 12 hours later. All blood samples for cardiac enzyme analysis were centrifuged, the serum separated and analysed at the time received to simulate the provision of a routine clinical service.

Creatine kinase activity was measured at $37^{\circ} \mathrm{C}$ with a Beckman CX7 analyser (Beckman Instruments, High Wycombe, UK) using commercially available reagents (Randox, Dublin, Republic of Ireland). The between batch imprecision of this method was $4 \cdot 1 \%$ at $147 \mathrm{IU} / 1$ and $2 \cdot 3 \%$ at $467 \mathrm{IU} / 1$. The laboratory upper reference range for $\mathrm{CK}$ was $195 \mathrm{IU} / 1$ for men and $170 \mathrm{IU} / 1$ for women.

Lactate dehydrogenase activity was measured using the same instrument and reagents from the same supplier. The between batch imprecision of this method was $1 \cdot 6 \%$ at 354 
Table 1 Time from onset of chest pain until admission to hospital ${ }^{*}$

\begin{tabular}{lcl}
\hline $\begin{array}{l}\text { Time from onset } \\
\text { of chest pain }\end{array}$ & AMI & $\begin{array}{l}\text { Other causes } \\
\text { of chest pain }\end{array}$ \\
\hline Less than six hours & 27 & 44 \\
Six to 12 hours & 6 & 21 \\
More than 12 hours & 4 & 30 \\
\hline
\end{tabular}

*Significantly less $(p<0.001)$ for patients with myocardial infarction compared with the other diagnostic groups.

Table 2 Discharge diagnosis of patients over 65 years of age following admission with acute chest pain

\begin{tabular}{lc}
\hline Diagnostic category & Number of patients \\
\hline Angina pectoris & 63 \\
Myocardial infarction & 41 \\
Gastrointestinal disease & 11 \\
$\quad$ reflux oesophagitis & 5 \\
hiatus hernia & 2 \\
gastritis & 1 \\
dysphagia & 1 \\
oesophageal ulceration & 1 \\
gastrointestinal haemorrhage & 1 \\
Angina pectoris plus dysrhythmia & 10 \\
Angina pectoris plus cardiac failure & 10 \\
Musculoskeletal pain & 5 \\
Pneumonia & 4 \\
Non-specific chest pain & 4 \\
Pulmonary emboli & 2 \\
Hypotension & 2 \\
Hypertension & 2 \\
Cerebrovascular accident & 1 \\
Micturition syncope & 1 \\
Adenocarcinoma with metastases & 1 \\
\hline
\end{tabular}

IU $/ 1$ and $1.0 \%$ at $923 \mathrm{IU} / 1$. The laboratory reference range for $\mathrm{LDH}$ was 230-460 IU/1.

The logarithm of the rate of change in CK activity was calculated from the change in $\mathrm{CK}$ activity between the admission sample and the second sample collected eight to 12 hours later, as described by Collinson et al. ${ }^{1}$ The logarithm of the change in CK activity was only calculated when one of the two CK values was greater than $170 \mathrm{IU} / 1$. If neither of the $\mathrm{CK}$ values in the timed samples were greater

Figure 1 Peak serum $C K$ activity in samples collected during hospital stay.

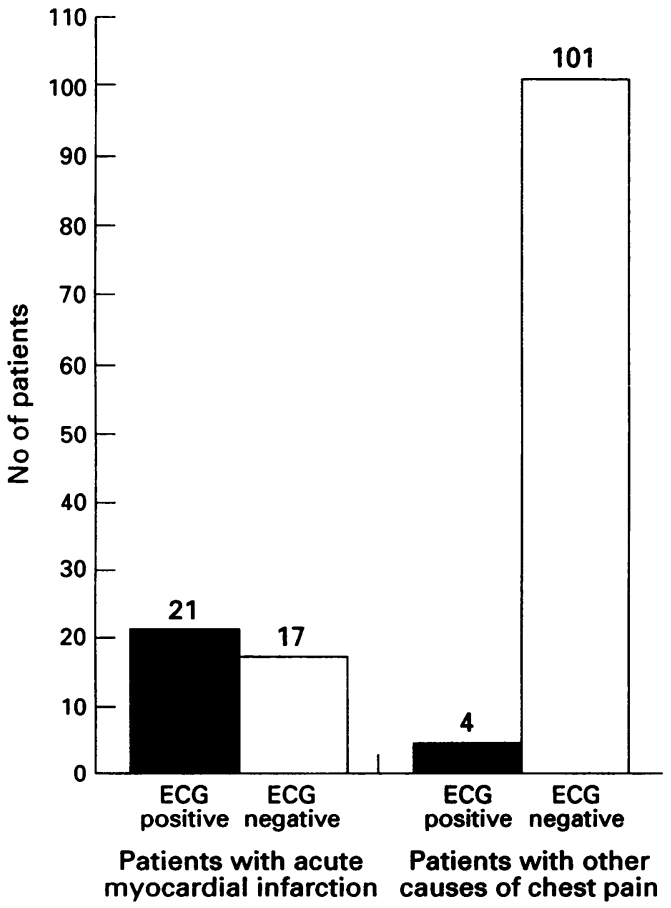

than $170 \mathrm{IU} / \mathrm{l}$, the patient was classified as having a normal timed CK result. A logarithm of the rate of change of $\mathrm{CK}$ activity/hour over 0.015 was considered to be a positive result indicative of $\mathrm{AMI} .^{1}$

The electrocardiographic criteria used for the diagnosis of AMI were ST elevation, T wave inversion, and the development of $Q$ waves.

Distribution of results within patient groups was compared using the Wilcoxon sign rank test, means were compared using the Student's $t$ test, and the distribution of results between groups compared using the $\chi^{2}$ test.

\section{Results}

A final diagnosis of AMI was made in 41 ( $26 \% ; 18$ women) of the 156 patients studied. The mean (SEM) age of those who sustained an AMI was $76(6.5)$ years compared with $75.5(7 \cdot 2)$ years $(p=0.611)$ for those who had not.

Patients had difficulty recalling the exact time of onset chest pain. Estimation of the duration of chest pain before admission was only possible for 132 patients. Most of the patients who had sustained an AMI presented less than six hours after the onset of chest pain compared with more than six hours for those who had not $(\mathrm{p}<0.001)$ (table 1$)$.

The final diagnosis recorded at discharge was made on the basis of clinical presentation, clinical course, CK and $\mathrm{LDH}$ values during the three days following admission, and serial ECGs. Myocardial infarction was the second most frequent diagnosis with angina pectoris the most common (table 2). Other diagnoses included pneumonia, musculoskeletal pain, and gastrointestinal causes of lower chest pain (table 2).

Peak CK activity in samples from patients who had sustained an infarction overlapped considerably with that in those who had another cause of chest pain (fig 1). The sensitivity of the peak CK value for the diagnosis of AMI was $97.5 \%$ and the specificity $83 \%$, giving a predictive value of $99 \%$ for the exclusion but only $68 \%$ for a diagnosis of AMI.

Peak LDH activity contributed little to the diagnosis. The sensitivity of a peak LDH value greater than the reference range for AMI was $100 \%$, but the specificity was only $47 \cdot 8 \%$. This gave a predictive value of $100 \%$ for a normal result but of only $40 \%$ for a raised $\mathrm{LDH}$ result.

When the admission ECGs were examined, there were no signs of AMI in 17 of the 41 patients later diagnosed as having sustained an AMI (fig 2). The sensitivity of the admission ECG, therefore, was only $55 \%$, although the specificity was $96 \%$. The predictive value of a normal admission ECG for the exclusion of AMI was $86 \%$ and was $84 \%$ for an ECG with changes consistent with AMI.

Calculation of the logarithm of the rate of change of serum CK activity between the samples collected on admission and eight to 12 hours later clearly separated the patients whose discharge diagnosis was AMI from 
Figure 2 Number of patients with changes on admission ECG diagnosed as having an $A M I$ compared with those with other causes of chest pain.

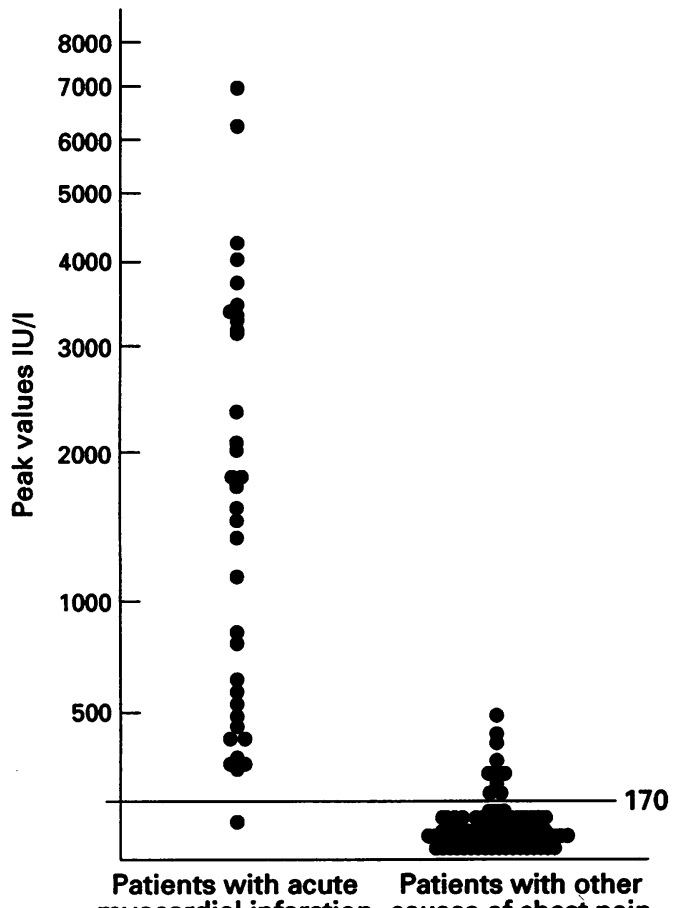

myocardial infarction causes of chest pain

those whose chest pain resulted from other causes. There were three apparently false negative results. One because the patient had infarcted following admission to hospital; this occurred after both timed CK samples had been collected.

There were two false positive results. On examination of the case notes, these patients probably sustained an AMI, a diagnosis based on standard ECG and cardiac enzyme results, and clinical history. If the false positive and negative results were included then the timed samples for CK analysis had a sensitivity of $93 \%$ and a specificity of $98 \%$ for the detection

Figure 3 Slope change in CK activity/hour in patients with $A M I$ compared with those with other causes of chest pain. The black bar represents patients whose CK activity did not rise above $170 \mathrm{IU} / \mathrm{l}$ between the first two samples and whose $C K$ slope activity was not analysed.

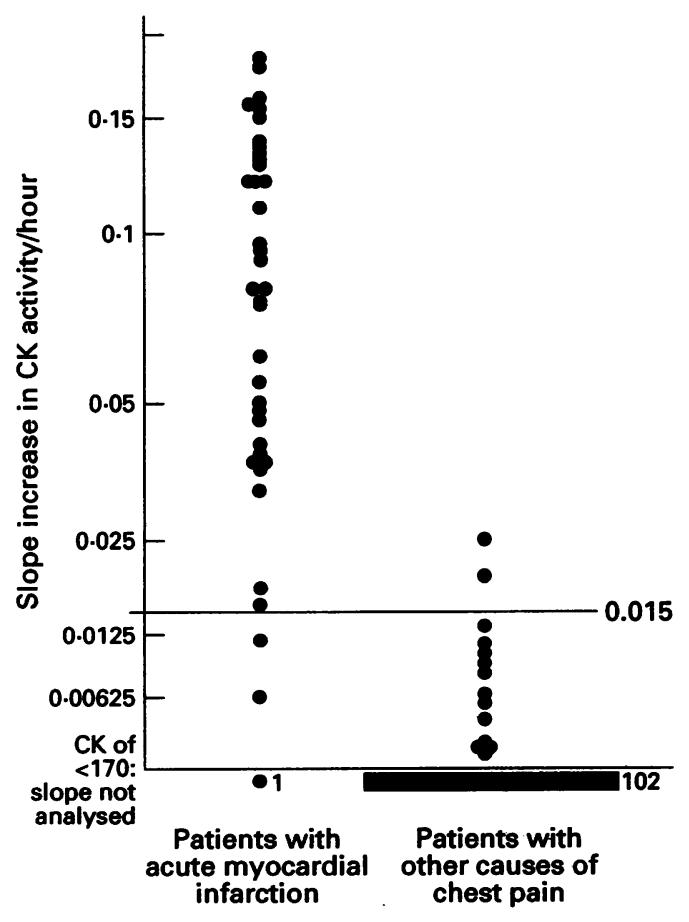

of AMI. This resulted in a predictive value of $97 \%$ for excluding and $95 \%$ for confirming a diagnosis of AMI, with the misclassification of only five (3\%) patients within 12 hours of admission. If the three misclassified patients were excluded, the predictive value of a negative timed CK result was $99 \%$ and that of a positive result was $100 \%$.

\section{Discussion}

This study has shown that the collection of two timed samples for $\mathrm{CK}$ analysis and the calculation of the rate of change of CK activity/hour can be used to confirm a diagnosis of AMI within 12 hours of admission.

The differences in the predictive value of the timed CK analysis between this study and the others may be explained by differences in the illnesses present in the population studied. The small number of coronary care unit patients $(\mathrm{n}=30)$ originally studied by Collinson et $a l^{1}$ may explain why they achieved better predictive values. The larger number of coronary care unit patients $(n=$ 101) studied by Vijan et $a l$ may explain the lower predictive value of a negative result.

All patients over 65 years of age presenting with acute chest pain were eligible for inclusion in this study. Therefore, more patients were studied $(n=156)$ and a smaller proportion sustained an AMI (26\% compared with $53 \%$ in the study by Collinson et al ${ }^{1}$ and $43 \%$ in that of Vijan et $a l^{7}$ ). A larger number of patients had confounding diagnoses $(n=115)$ and, therefore, the results of this study may represent the performance of timed CK analysis in routine clinical practice more accurately.

Timed CK samples do not confirm the diagnosis of AMI sufficiently rapidly to influence treatment with thrombolytic agents. Confirmation of the diagnosis within 12 hours of admission, however, will influence subsequent patient management and could lead to more efficient use of beds in coronary care units. ${ }^{5}$

Determination of the concentrations of Troponin $t$, myoglobin, and the $M M$ and $M B$ isoenzymes of CK (CK-MM and CK-MB, respectively) has been proposed as a method of confirming a diagnosis of AMI. ${ }^{9-13}$ None of the above tests are suitable for the early diagnosis of AMI and, therefore, do not influence the decision to administer thrombolytic treatment. Difficulties with these tests include non-specificity and the need for dedicated equipment. Accurate timing of the collection of single samples in relation to the onset of chest pain for the measurement of the CK$M B$ concentration, and CK-MM and CK$\mathrm{MB}$ isoforms may be difficult. In this study patients found it difficult to recall exactly the onset of their chest pain.

Current evidence suggests that thrombolytic treatment is most effective when administered as soon as possible after the onset of chest pain, therefore, any delay could impair patient outcome. Laboratory tests for AMI will probably continue to be used to confirm diagnosis and for subsequent patient 
management, but will not influence the decision to administer thrombolytic treatment. Timed sequential analysis of CK activity has one disadvantage in that two samples are required; however, this test is more specific, does not need specialised equipment, and may be provided on a 24 hour basis. The false positive and false negative results produced by timed CK analysis support the recommendation of Collinson et al ${ }^{1}$ that this test should be used in association with a confirmatory test, such as CK-MB analysis. Earlier confirmation can be achieved if slope analysis is used in association with one of the other sensitive early tests, such as determination of the myoglobin concentration or analysis of the CK$\mathrm{MM}$ or $\mathrm{CK}-\mathrm{MB}$ isoforms.

We thank the physicians of the Wirral Hospital Trust for allowing us access to their patients.

1 Collinson PO, Rosalki SB, Flather M, Wolman R, Evans $T$. Early diagnosis of myocardial infarction by timed sequential enzyme measurements. Ann Clin Biochem 1988;25:376-82.

2 Collinson PO, Ramhamadamy EM, Rosalki SB, Joffe J, Evans DH, Fink RS, et al. Diagnosis of acute myocardial infarction from sequential enzyme measurements obtained within 12 hours of admission to hospital. $f$ Clin Pathol 1989;42:1126-31.
3 Rutty G, Patel S, O'Gorman P. Application of the regression coefficient to timed serial CK measurements in the early diagnosis of myocardial infarction. Ann Clin Biochem 1986;26:558-9.

4 Bernstein LH, Reynoso G. Creatine kinase B-subunit activity in serum in cases of suspected myocardial infarction-a predictive model based on the slope of $\mathrm{MB}$ increase and percentage CK-MB activity. Clin Chem 1983;29:590-2.

5 Collinson PO, Ramhamadamy EM, Stubs PJ, Rosalki SB, Garrett HM, Mosley D, et al. Rapid enzyme diagnosis of patients with acute chest pain reduces patient stay in the coronary care unit. Ann Clin Biochem 1993;30:17-22.

6 Johnson JD, Collinson PO, Rosalki SB. MIDAS: Myocardial infarct diagnosis by assessment of slope. Ann Clin Biochem 1993;30:407-9.

7 Vijan SG, Ingle AR, Hill PG, Millar-Craig MW. Failure of creatine kinase slope values to reliably predict acute
myocardial infarction. Ann Clin Biochem 1991;28:103-4.

myocardial infarction. Ann Clin Biochem 1991;28:103-4.
8 Dufour DR, LaGrenade A, Guerra J. Rapid serial enzyme measurements in evaluation of patients with suspected myocardial infarction. Am $\mathcal{F}$ Cardiol 1989;63:652-5.

9 Collinson PO, Moseley D, Stubbs PJ, Carter CD. Troponin $T$ for the differential diagnosis of ischaemic myocardial damage. Ann Clin Biochem 1993;30:11-6.

10 Chepelle J, Heusghem C. Semi-quantitative estimation of serum myoglobin by a rapid latex agglutination method: an emergency screening test for acute myocardial infarction. Clin Chem Acta 1985;145:143-50.

11 Kanemitsu F, Okigaki T. Creatine kinase MB isoforms for early diagnosis and monitoring of acute myocardial infarction. Clin Chim Acta 1992;206:191-9.

12 Wu AHB, Garnet TG, Wu VH, Brockie RE, Nshikawa A. Early diagnosis of acute myocardial infarction by rapid analysis of creatine kinase isoenzyme-3 (CK-MM) subtypes. Clin Chem 1987;33:358-62.

13 Collinson PO, Rosalki SB, Kuwana T, Garrett HM, Ramhamadamy EM, Baird IM, et al. Early diagnosis of acute myocardial infarction by CK-MB mass measurement. Ann Clin Biochem 1992;29:43-7. 